\title{
Multibody Modeling and Balance Control of a Reaction Wheel Inverted Pendulum Using LQR Controller
}

\author{
Ümit Önen a,1, Abdullah Çakan b,2,* \\ a Department of Mechatronics Engineering, Necmettin Erbakan University, Konya, Turkey \\ ${ }^{\mathrm{b}}$ Department of Mechanical Engineering, Konya Technical University, Konya, Turkey \\ 1 uonen@erbakan.edu.tr; ${ }^{2}$ acakan@ktun.edu.tr \\ * Corresponding Author
}

ARTICLE INFO

Article history

Received 27 March 2021

Revised 01 April 2021

Accepted 03 April 2021

Keywords

Reaction wheel pendulum;

Inverted pendulum;

LQR controller;

Multibody dynamics;

Matlab;

Simulink

\begin{abstract}
In this study, modeling and LQR control of a reaction wheel inverted pendulum system is described. The reaction wheel inverted pendulum model is created by using a 3D CAD platform and exported to Simscape Multibody. The multibody model is linearized to derive a state-space representation. A LQR (Linear-quadratic regulator) controller is designed and applied for balance control of the pendulum. The results show that deriving a state-space representation from multibody is an easy and effective way to model dynamic systems and balance control of the reaction wheel inverted pendulum is successfully achieved by LQR controller. Results are given in the form of graphics.
\end{abstract}

This is an open access article under the CC-BY-SA license.

\section{Introduction}

Researchers have been studying the development and implementation of control methods for countless years. Inverted pendulum systems are commonly studied in control systems to compare control methods as a benchmark problem. There are many different types of inverted pendulum such as rotary, multiple links, flexible and inertia (reactional) wheel inverted pendulum systems [1-4]. In this research, a reactional wheel inverted pendulum system is considered [5]. A reaction wheel is a flywheel that is used in many systems such as satellite attitude controls and driving some kind of robots [6, 7]. The Reaction wheel inverted pendulum is a pendulum system consists of a rotating wheel on top and a simple inverted pendulum rotating about a pinned joint. Also, many control techniques are studied using these different types of inverted pendulum systems such as PID (proportional-integral-derivative), LQR (linear-quadratic regulator), Fuzzy Logic and Sliding Mode controllers [8-11]. LQR controller is a state feedback controller and it is used to control linear systems [12]. Hence, in this study, a reaction wheel inverted pendulum system is created by using a 3D CAD platform, exported to Simscape Multibody which is a toolbox of Matlab/Simulink to simulate dynamics systems and state-space representation is derived from the multibody model and a full-state feedback LQR controller is applied to balance the pendulum by using Matlab/Simulink. 


\section{Modeling of Reaction Wheel Inverted Pendulum}

In this section modeling of the reaction wheel inverted pendulum is described. Model of reaction wheel inverted pendulum is created by using a 3D CAD platform and exported to Simscape Multibody which is a toolbox of Matlab/Simulink to simulate systems and state-space representation is derived from the multibody model. Designed reaction wheel inverted pendulum system shown in Fig. 1 and the system parameters shown in Table 1.

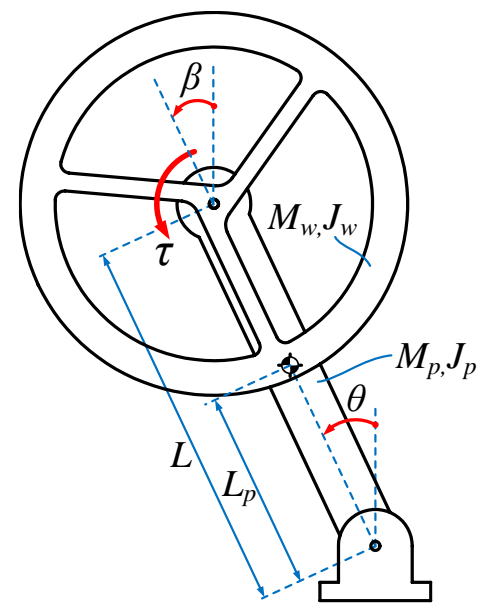

Fig. 1. Reaction wheel inverted pendulum system

Table 1. Reaction wheel inverted pendulum system parameters

\begin{tabular}{ccc}
\hline Symbol & Description & Value and unit \\
\hline$M_{w}$ & Mass of reaction wheel & $0.554 \mathrm{~kg}$ \\
$M_{p}$ & Mass of pendulum & $0.141 \mathrm{~kg}$ \\
$J_{w}$ & Wheel mass moment of inertia & $4.36 \times 10^{-3} \mathrm{kgm}^{2}$ \\
$J_{p}$ & Pendulum mass moment of inertia & $0.69 \times 10^{-3} \mathrm{kgm}^{2}$ \\
$L$ & Pendulum length & $0.21 \mathrm{~m}$ \\
$L_{p}$ & Pendulum center of mass length & $0.11 \mathrm{~m}$ \\
$\tau$ & Control torque & Nm \\
$\theta$ & Pendulum angle & degree \\
$\beta$ & Wheel angle & degree \\
\hline
\end{tabular}

In the reaction wheel inverted pendulum system modeling, the input of the system is torque $\tau$ that applied to the reaction wheel, the outputs are pendulum angle $\theta$ and wheel angle $\beta$. The pendulum and reaction wheel are assumed to be rigid. Created Simscape Multibody model shown in Fig. 2. This model is linearized using the Matlab platform and the state-space representation of the system was obtained to be used in LQR control studies of this paper. State matrix $A$, input matrix $B$, output matrix $C$ and feedforward matrix $D$ of the state-space representation are shown (1) and (2).

$$
\begin{aligned}
A & =\left[\begin{array}{cccc}
0 & 1 & 0 & 0 \\
44.2924 & 0 & 0 & 0 \\
0 & 0 & 0 & 1 \\
-44.2924 & 0 & 0 & 1
\end{array}\right] \quad B=\left[\begin{array}{c}
0 \\
-38.5631 \\
0 \\
268.0902
\end{array}\right] \\
C & =\left[\begin{array}{llll}
1 & 0 & 0 & 0 \\
0 & 1 & 0 & 0 \\
0 & 0 & 1 & 0 \\
0 & 0 & 0 & 1
\end{array}\right] \quad D=\left[\begin{array}{l}
0 \\
0 \\
0 \\
0
\end{array}\right]
\end{aligned}
$$





Fig. 2. Simscape Multibody model of reaction wheel inverted pendulum system

\section{LQR Control of Reaction Wheel Inverted Pendulum}

Linear Quadratic Regulator (LQR) is a full-state feedback control technique that is suitable to control reaction wheel inverted pendulum system. LQR Control block diagram of reaction wheel inverted pendulum system shown in Fig. 3.

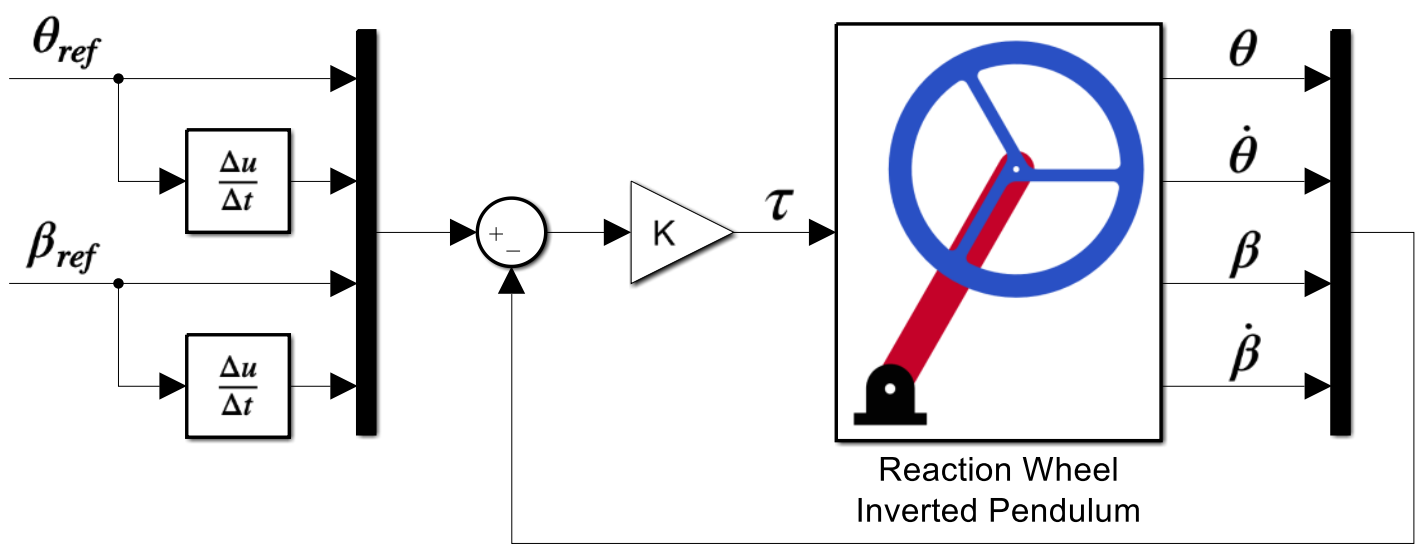

Fig. 3. LQR Control block diagram of reaction wheel inverted pendulum system

Equations of motion of the system can be described in the form (3) in this equation $A$ and $B$ are the state and input matrices of the system, the LQR determines the control signal $u$ to minimize of objective function $J$ shown in (4).

$$
\begin{aligned}
& \dot{x}=A x+B u \\
& J=\int_{0}^{\infty}\left(x_{r e f}-x(t)\right)^{T} Q\left(x_{r e f}-x(t)\right)+u(t)^{T} R u(t) d t
\end{aligned}
$$

The matrices $Q$ and $R$ remove the error between desired input and the control response. In this paper state vector $x$ defined in (5) and $A$ and $B$ matrices are used in LQR controller design. The next step in control design is to find the vector of state-feedback control gains $K$ matrix. $K$ is determined using Matlab platform with "Iqr" command with $A, B, Q$ and $R$ matrices. $Q$ and $R$ matrices are obtained by trial and error and shown in (6). The command is used in the form of (7) and $K$ is determined. Determined $K$ matrix is shown in (8). 


$$
\begin{aligned}
x & =\left[\begin{array}{llll}
\theta & \dot{\theta} & \beta & \dot{\beta}
\end{array}\right]^{T} \\
Q & =\left[\begin{array}{cccc}
10^{4} & 0 & 0 & 0 \\
0 & 1 & 0 & 0 \\
0 & 0 & 1 & 0 \\
0 & 0 & 0 & 1
\end{array}\right], \quad R=1 \\
K & =\operatorname{lqg}(A, B, Q, R) \\
K & =-\left[\begin{array}{lllll}
147.6226 & 17.6263 & 1 & 1.4530
\end{array}\right]
\end{aligned}
$$

\section{Results and Discussion}

While reaction wheel inverted pendulum system is controlled, desired pendulum angle is 0 degree and desired wheel rotational position is 0 degree choses. LQR controlled pendulum angle and pendulum angular velocity response shown in Fig. 4 and Fig. 5 respectively.

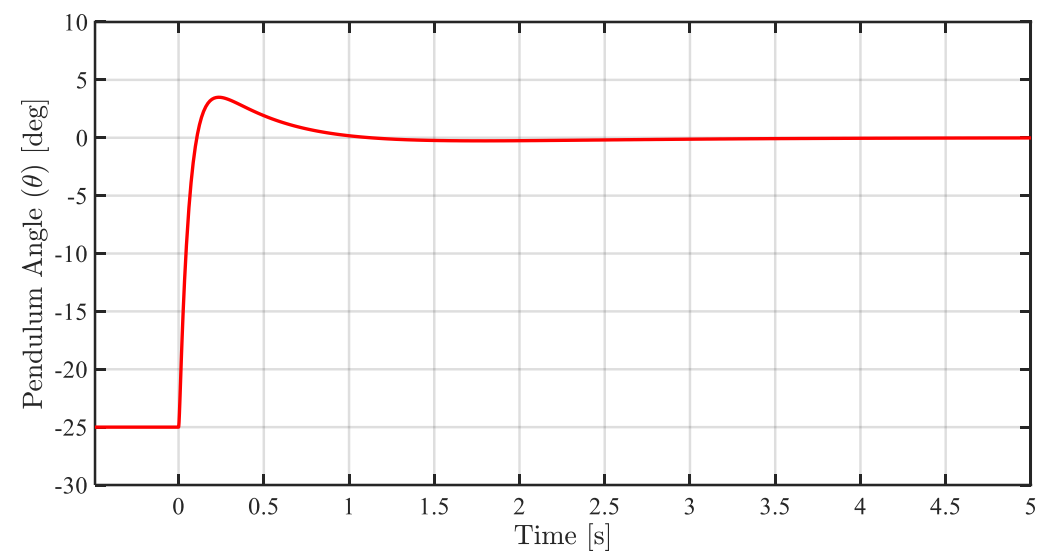

Fig. 4. Pendulum angle response of the system

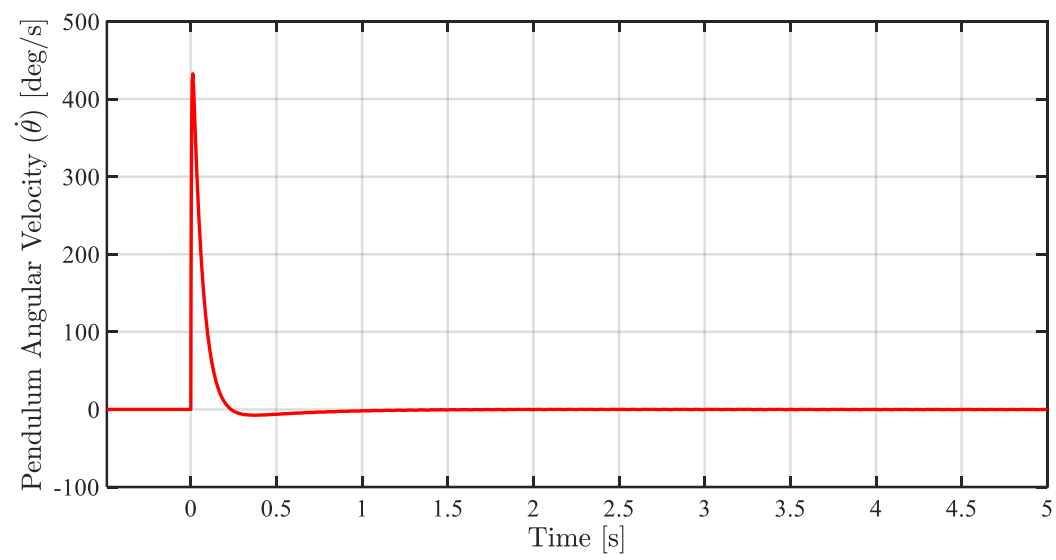

Fig. 5. Pendulum angular velocity response of the system

Moreover, wheel angle and wheel angular velocity of the reaction wheel inverted pendulum system is given in Fig. 6 and Fig. 7 respectively. In addition, LQR controlled torque change is given in Fig. 8. It can be said pendulum angle and wheel angle are reached to desired values. As a result, pendulum angle and wheel angle of the system are achieved successfully using LQR controller. As a result, designed LQR (Linear-quadratic regulator) controller can be used effectively in linearized systems. In order to see the results clearly time axis of the figures is widened. 


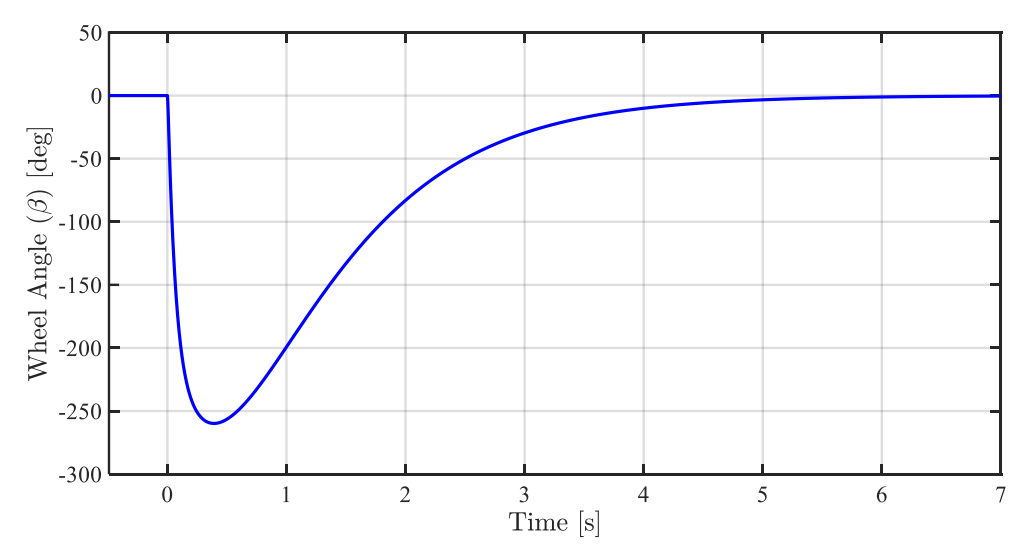

Fig. 6. Reaction wheel angle response of the system

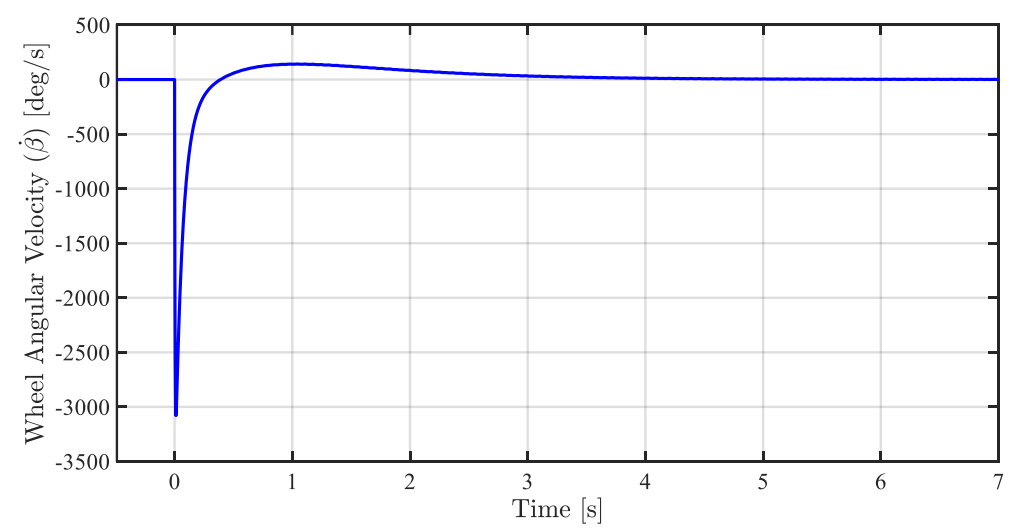

Fig. 7. Reaction wheel angular velocity response of the system

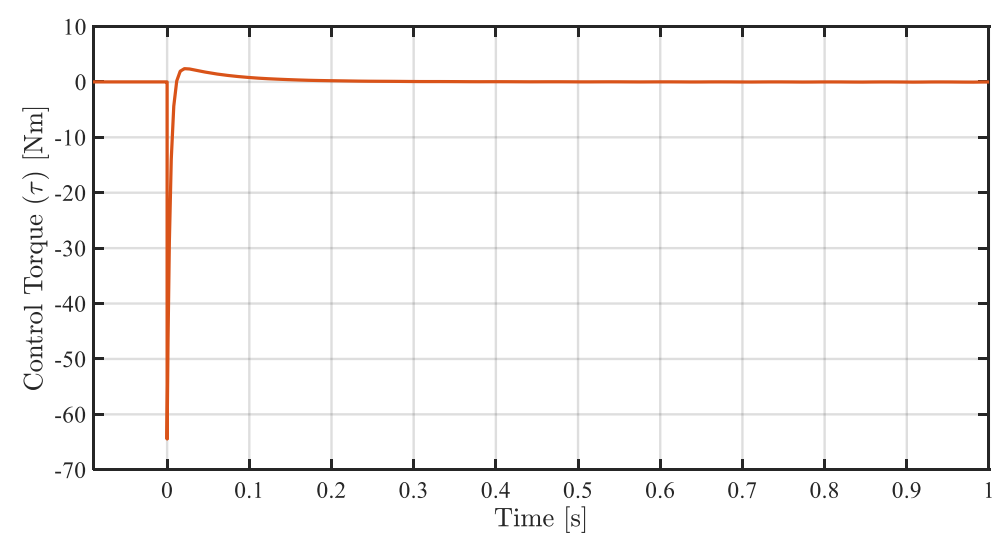

Fig. 8. Control torque for the balance the system -25 degrees to 0

\section{Conclusion}

The paper presents modeling and LQR control of a reaction wheel inverted pendulum system. 3D CAD platform is used for modeling and Matlab is used for both linearization and controller design. Thus, these types of mechanical systems can be modeled and controlled without a mathematical model by using two different engineering platforms. A LQR controller is designed and applied in Matlab/Simulink. LQR controller $\mathrm{Q}$ and $\mathrm{R}$ matrices are determined by the trial-error method. The controller is successfully achieved desired pendulum position. According to the results, the multibody modeling method is an effective and easy way to 
modeling the control systems. For better results, different optimization algorithms can be used to determine $\mathrm{Q}$ and $\mathrm{R}$ matrixes or different control techniques can be used.

\section{References}

[1] M. Akhtaruzzaman and A. A. Shafie, "Modeling and control of a rotary inverted pendulum using various methods, comparative assessment and result analysis," in 2010 IEEE International Conference on Mechatronics and Automation, 2010, IEEE, pp. 1342-1347. https://doi.org/10.1109/ICMA.2010.5589450.

[2] D. J. Block, K. J. Åström, and M. W. Spong, "The reaction wheel pendulum," Synthesis Lectures on Control and mechatronics, vol. 1, no. 1, pp. 1-105, 2007. https://doi.org/10.2200/S00085ED1V01Y200702CRM001.

[3] K. Furuta, T. Okutani, and H. Sone, "Computer control of a double inverted pendulum," Computers \& Electrical Engineering, vol. 5, no. 1, pp. 67-84, 1978. https://doi.org/10.1016/00457906(78)90018-6.

[4] J. Tang and G. Ren, "Modeling and simulation of a flexible inverted pendulum system," Tsinghua Science and Technology, vol. 14, no. S2, pp. 22-26, 2009. https://doi.org/10.1016/S10070214(10)70025-0.

[5] G. Belascuen and N. Aguilar, "Design, modeling and control of a reaction wheel balanced inverted pendulum," in 2018 IEEE Biennial Congress of Argentina (ARGENCON), 2018: IEEE, pp. 1-9. https://doi.org/10.1109/ARGENCON.2018.8646093.

[6] Z. Ismail and R. Varatharajoo, "A study of reaction wheel configurations for a 3-axis satellite attitude control," Advances in Space Research, vol. 45, no. 6, pp. 750-759, 2010. https://doi.org/10.1016/j.asr.2009.11.004.

[7] F. Zhang, P. Washington, and D. A. Paley, "A flexible, reaction-wheel-driven fish robot: Flow sensing and flow-relative control," in 2016 American Control Conference (ACC), 2016: IEEE, pp. 1221-1226. https://doi.org/10.1109/ACC.2016.7525084.

[8] A. Çakan and Ü. Önen, "Dynamic Modelling and Control of a Reaction Wheel Inverted Pendulum Using MSC Adams And MATLAB," in X International Conference Industrial Engineering and Environmental Protection 2020 (IIZS 2020), Zrenjanin, Serbia, 2020 2020, 2020, pp. 78-82. https://www.researchgate.net/publication/344783742

[9] B. H. Nguyen, M. P. Cu, M. T. Nguyen, M. S. Tran, and H. C. Tran, "LQR and Fuzzy Control for Reaction Wheel Inverted Pendulum Model," Robotica \& Management, vol. 24, no. 1, pp. 19-23, 2019. http://robotica-management.uem.ro/index.php?id=704

[10] I. Siradjuddin, E. Pradani, E. Rohadi, S. Adhisuwignjo, M. Kusumawardani, and I. Fitriani, "Designing, implementing and analysing optimal controllers on a non-linear reaction wheel pendulum," in Journal of Physics: Conference Series, 2019, vol. 1402, no. 4: IOP Publishing, p. 044025. https://doi.org/10.1088/1742-6596/1402/4/044025.

[11] N. Sun, Y. Fang, and H. Chen, "A novel sliding mode control method for an inertia wheel pendulum system," in 2015 International Workshop on Recent Advances in Sliding Modes (RASM), 2015: IEEE, pp. 1-6. https://doi.org/10.1109/RASM.2015.7154585.

[12] J. P. Hespanha, "LQG/LQR controller design," Undergraduate Lecture Notes, University of California, Santa Barbara, California, USA, 2007. 